\title{
Rehabilitation of Hemi-Mandibulectomy Patient with Twin Occlusion Prosthesis
}

\author{
Pramod K Chahar ${ }^{1}$, Abir Sarkar², E Mahesh Gowda ${ }^{3}$, Poonam Prakash ${ }^{4}$, Vijaya Kumar R ${ }^{5}$, Prashant Awasthi ${ }^{6}$ \\ ${ }^{1}$ PG Resident, Department of Dental Surgery and Oral Health Sciences, ${ }^{2}$ PG Resident, Department of Dental Surgery and \\ Oral Health Sciences, ${ }^{3}$ Associate Professor, Department of Dental Surgery and Oral Health Sciences, ${ }^{4}$ Assistant Professor, \\ Department of Dental Surgery and Oral Health Sciences, ${ }^{5}$ Assistant Professor, Department of Dental Surgery and Oral Health \\ Sciences, ${ }^{6}$ Assistant Professor, Department of Dental Surgery and Oral Health Sciences, Armed Forces Medical College, Pune, \\ Maharashtra, India
}

Corresponding author: Pramod K Chahar, Department of Dental Surgery and Oral Health Sciences, Armed Forces Medical College, Pune, 411040, Maharashtra, India

DOI: http://dx.doi.org/10.21276/ijcmsr.2019.4.1.6

How to cite this article: Pramod K Chahar, Abir Sarkar, E Mahesh Gowda, Poonam Prakash, Vijaya Kumar R, Prashant Awasthi. Rehabilitation of hemi-mandibulectomy patient with twin occlusion prosthesis. International Journal of Contemporary Medicine Surgery and Radiology. 2019;4(1):A24-A26.

\section{A B S T R A C T}

Introduction: The mandible is a single bone that makes the lower third of the face and forms peripheral boundaries of floor of the mouth. Surgical resection of mandible owing to benign, malignant neoplasm, osteoradionecrosis is common. Loss of mandibular continuity causes deviation and rotation inferiorly due to muscle pull and scar contracture affecting mandibular movements, mastication, speech and aesthetics.

Case Report: This case report describes prosthetic rehabilitation of a patient with post hemi-mandibulectomy residual defect. Patient underwent surgical resection of mandible right side distal to canine resulted in deviation and rotation of mandible towards the defect side. Because of restricted manipulation of mandible towards non-resected side, dual occlusion prosthesis in maxillary arch was planned to improve masticatory efficiency.

Conclusion: Patients with excessive scar contracture limits mandibular manipulation towards normal side to achieve occlusion. These patients are rehabilitated with dual occlusion prosthesis to restore function.

Keywords: Rehabilitation of Hemi-Mandibulectomy, Twin Occlusion Prosthesis

\section{INTRODCUTION}

The mandible is an important structure that along with muscles of mastication, forms the lower third of the face. It forms the peripheral boundaries of oral cavity and floor of the mouth. Muscles of mastication are attached to the mandible bilaterally, which plays an important role in various function like speech, mastication, swallowing and respiration. Disruption of mandible either because of trauma or surgical resection owing to benign, malignant neoplasm or osteoradionecrosis is common. The resection of mandible can be total or segmental with or without loss of continuity depending on the extension of the lesion. ${ }^{1}$ Resection of the mandible may cause topographic defects while maintaining the continuity but facial form and movements of the mandible can often be preserved. Loss of mandibular continuity causes deviation of remaining mandibular segment towards the resected side and rotation inferiorly due to muscle pull and scar contracture affecting mandibular movements, mastication, speech and aesthetics. Mandibulectomy with radical neck dissection increases this deviation which results in facial disfigurement, loss of occlusal contact, in many cases, loss of lip competency for saliva control and to initiate the swallowing process. ${ }^{2,3}$
Literature shows various techniques to correct mandibular deviation and to regain form and function. It can vary from intermaxillary fixation with elastics, palatal or mandibular guiding flange prosthesis anchored on natural teeth or the dual occlusion prosthesis where manipulation of mandible to achieve occlusion on normal side is not possible because of excessive deviation caused due to severe scar contracture and fibrosis of remaining tissues. ${ }^{4}$ Surgical reconstruction is always not possible in all cases, in those cases prosthetic rehabilitation plays a very important role to regain form and function. This article describes fabrication of dual occlusion prosthesis for maxillary arch in hemi-mandibulectomy patient to achieve occlusion on normal side which improves masticatory efficiency and hence enhances the quality of life.

\section{CASE REPORT}

A 55 years old male patient was reported to the division of prosthodontics and crown and bridge of tertiary care hospital with the history of squamous cell carcinoma right side of mandible. Three months back patient underwent hemi-mandibulectomy and modified radical neck dissection followed by radiation therapy for one month at $50 \mathrm{~Gy}$. Patient's chief complaints were difficulty in mastication and speech 
with restricted mouth opening. Extra-oral examination of the patient revealed gross facial asymmetry with deviation of mandible towards the right side and inferior rotation of the mandible (Fig 1a). Intra oral examination revealed partially edentulous maxillary arch with missing 18,17,16,26,28, root stumps 14,24 and Ellis class II fractured 11, resected mandible distal to 43 (Fig 1b). Post radiation therapy mucositis and xerostomia was also present. Patient had reduced mouth opening to $11 \mathrm{~mm}$ which made it difficult to start with prosthetic rehabilitation. An orthopantomogram radiograph revealed resected mandible distal to left canine involving entire mandible of right side without condylectomy representing class II type postsurgical impairment according to Cantor and Curtis classification. ${ }^{5}$

Patient was referred for physiotherapy and subjected to mouth opening exercise by using ice cream sticks in incremental manner for 4 weeks. For management of xerostomia, patient was advised to use artificial saliva and topical fluoride application was done prevent caries. Instructions were given to maintain oral hygiene and to preserve periodontal health. After 4 weeks, patient was reviewed and adequate mouth opening of $28 \mathrm{~mm}$ was achieved, which was sufficient to start with impression procedures for prosthetic rehabilitation. Treatment options were surgical reconstruction or prosthetic rehabilitation or combination of both. Since patient had undergone radiation therapy, surgical reconstruction was not possible and also it was noted that guiding the mandible towards normal side to achieve occlusion was difficult due to excessive fibrosis of the tissue. So, the treatment objective was to restore function by improving mastication with the help of twin occlusion prosthesis in the maxillary arch.

Preliminary impressions were made in polyvinyl addition siloxane impression material (Affinis, Krupp Vacudent Co. Germany) of putty consistency after modification of plastic stock trays. Impressions were poured in type 3 dental stone (Kalabhai utrastone Type III, Mumbai) and casts were obtained. A heat-cure clear acrylic resin (DPI Heat cure acrylic resin, Mumbai, India) base was fabricated on maxillary arch and adams clasp and pin heads made of 20-gauge wire were incorporated to provide retention to the base. Occlusal rim of base plate wax (Maarc, Shiva products, Maharashtra, India) (Fig. 2a) was added to the palatal side of maxillary teeth up to the height of occlusal surfaces of teeth. Denture base along with occlusal rim was placed in patient's mouth and bite registration was done (Fig 2b). Bite was transferred to mean value articulator and articulation was done. Acrylic teeth (Acry Rock acrylic teeth, Jodhpur, India) were arranged on palatal side of maxillary teeth of non-resected side (Fig $3 a)$. This arrangement produced dual rows of teeth on non-resected side of mandible helped in achieving stable occlusion and improving masticatory efficiency. Try-in was done and after minor modifications and achieving satisfactory occlusion, denture base was processed, finished and polished (Fig 3b). Prosthesis was inserted in patients mouth and checked for occlusion (Fig $4 \mathrm{a}$ and $4 \mathrm{~b}$ ). Post insertion instructions were given and patient was recalled after every month, which showed marked improvement in the masticatory efficiency.

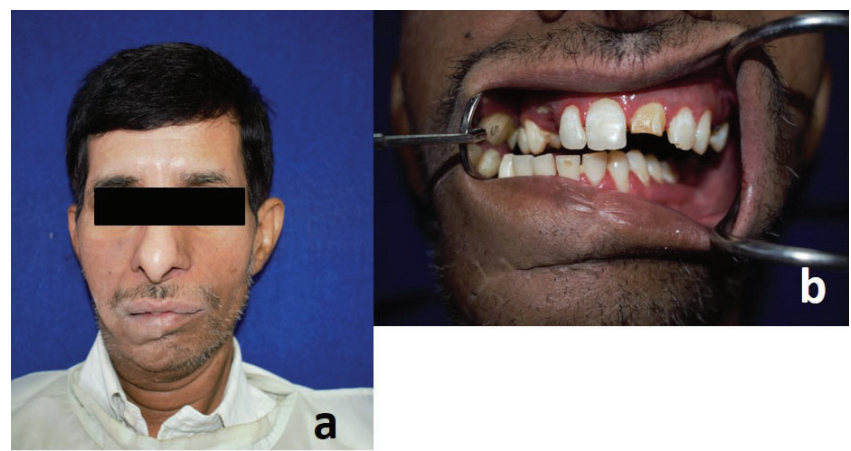

Figure-1: Pre-op (a) Extra oral (b) Intraoral

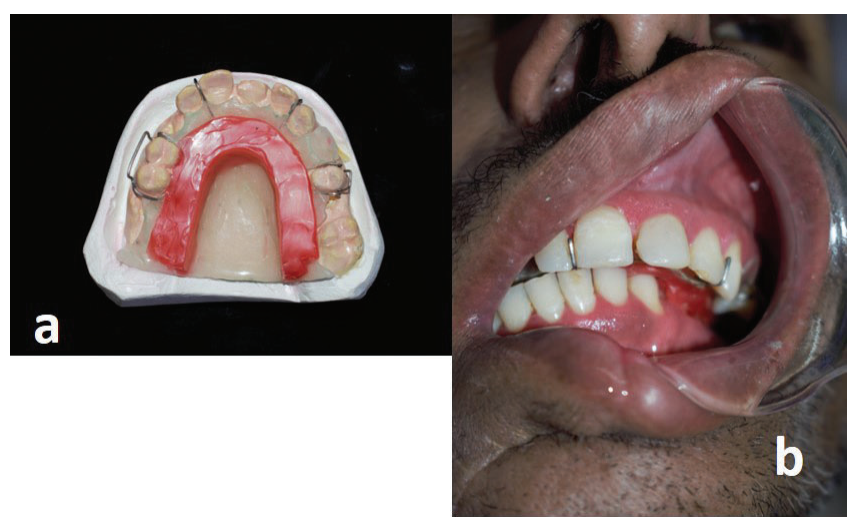

Figure-2: (a) Occlusal wax rim (b) Bite registration

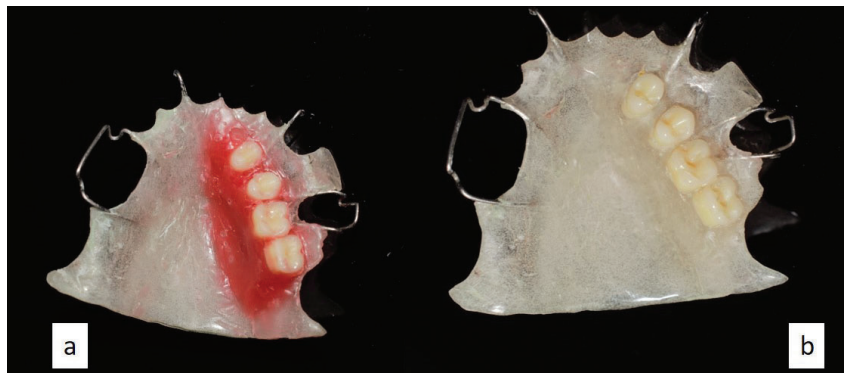

Figure-3: (a) Teeth arrangement (b) Processed prosthesis

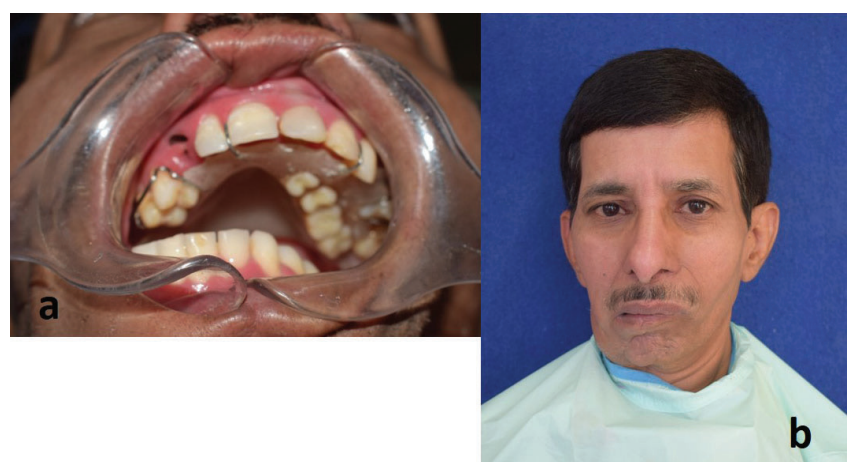

Figure-4: Post-op (a) Prosthesis in situ (b) Extra-oral

\section{DISCUSSION}

This functional rehabilitation of hemi mandibulectomy patient who has undergone resection without reconstruction is done by either guide flange or palatal ramp prosthesis which helps in guiding the mandible on non-resected side to achieve occlusion and hence mastication can be performed. ${ }^{3,6}$ Also, these prostheses reduce the facial asymmetry by 
preventing rotation and deviation of the mandible and hence improves aesthetics. In some patients due to excessive fibrosis of scar tissue and drawing of soft tissue to achieve primary closure after surgical resection of mandible, it becomes difficult to guide the mandible to achieve occlusal contacts on non- resected side. ${ }^{1}$ The patient in this case report, severe scar contracture and fibrosis of soft tissue along with radiation therapy resulted in limited guidance of mandible to the normal side. Hence a removable prosthesis with double rows of teeth that is dual occlusion was fabricated to improve masticatory efficiency. Another row of teeth palatal to natural teeth was arranged on normal side, so that patient could achieve occlusion with natural teeth on non -resected side. With this palatal row of teeth patient could masticate and natural teeth on buccal side supported the cheek and improved the aesthetics. Due to dual occlusion, functional rehabilitation of the patient was achieved. After one-week patient reported back with improved masticatory efficiency and quality of life.

\section{CONCLUSION}

The patients who have undergone mandibular resection without surgical reconstruction, the prognosis is poor and the rehabilitation is difficult because of deviation and rotation of mandible and restricted mouth opening. Guide flange prosthesis is most commonly used mode of prosthetic rehabilitation to guide the mandible in occlusion but in patients with difficult manipulation and severe deviation of mandible, it is not indicated. Dual occlusion prosthesis is a viable alternative to achieve functional rehabilitation in these patients.

\section{REFERENCES}

1. Beumer J, 3rd, Curtis TA, Marunick MT. St. Louis: Ishiyaku EuroAmerica; 1996. Maxillofacial Rehabilitation: Prosthodontic and Surgical Consideration; pp. 184-8.

2. Agarwal S, Praveen G, Agarwal SK, Sharma S. Twin occlusion: A solution to rehabilitate hemimandibulectomy patient-A case report. The Journal of Indian Prosthodontic Society. 2011;11(4):254

3. Chodankar K, Shingote S, Kajave M, Bandekar S. Twin Occlusion Prosthesis: An Alternative to Conventional Guide Ramp-A Case Report.

4. Taylor TD. Diagnostic considerations for prosthodontic rehabilitation of the mandibulectomy patient. In: Taylor TD, editor. Clinical Maxillofacial Prosthetics. Chicago: Quintessence Publishing; 2000. pp. 155-70

5. Cantor R, Curtis TA. Prosthetic management of edentulous mandibulectomy patients. Part I. Anatomic, physiologic, and psychologic considerations. Journal of Prosthetic Dentistry. 1971;25(4):446-57.

6. Sureja RA, Naveen YG, Sethuraman RA, Prajapati PA, PATEL JR. Twin occlusion prosthesis: a ray of hope for hemimandibulectomy patient-a case report. EJDTR. 2014;3(3):231-3.

Source of Support: Nil; Conflict of Interest: None

Submitted: 22-12-2018; Accepted: 20-01-2019; Published online: 05-02-2019 